\title{
Erratum to: Infectious Aspects and the Etiopathogenesis of Rheumatoid Arthritis
}

\author{
Michal Kasher Meron • Howard Amital • Daniel Shepshelovich • Ori Barzilai • \\ Maya Ram • Juan-Manuel Anaya • Roberto Gerli • Nicola Bizzaro • Yehuda Shoenfeld
}

Published online: 1 February 2014

(C) Springer Science+Business Media New York 2014

\section{Erratum to: Clinic Rev Allerg Immunol (2010) 38:287-291 DOI 10.1007/s12016-009-8158-6}

The original version of this article unfortunately contained a mistake. Professor Nicola Bizzaro's name appears as "Bizzaro Nicola" instead of "Nicola Bizzaro".

The correct name is shown in the author group and affiliation section.

The online version of the original article can be found at http://dx.doi.org/ 10.1007/s12016-009-8158-6.

M. K. Meron $\cdot$ H. Amital Department of Medicine 'D',

Meir Medical Center affiliated with Tel Aviv University Sackler

Faculty of Medicine,

Kfar-Saba, Israel

D. Shepshelovich $\cdot$ O. Barzilai $\cdot$ M. Ram $•$ Y. Shoenfeld $(\bowtie)$

Department of Internal Medicine B,

Center for Autoimmune Diseases affiliated with Tel Aviv

University Sackler Faculty of Medicine, Sheba Medical Center,

Tel Hashomer 52621, Israel

e-mail: shoenfel@post.tau.ac.il

\section{J.-M. Anaya}

Cellular Biology and Immunogenetics Unit,

Corporación para Investigaciones Biológicas,

Universidad del Rosario,

Medellin, Colombia

R. Gerli

Rheumatology Unit,

Department of Clinical and Experimental Medicine,

University of Perugia,

Perugia, Italy

N. Bizzaro

Laboratorio di Patologia Clinica,

Ospedale Civile,

30027 S Donà di Piave, Venice, Italy

Y. Shoenfeld

Laura Schwarz-Kipp, Tel Aviv University,

Tel Aviv, Israel 